\title{
Anatomic morphological study of thoracolumbar foramen in normal adults
}

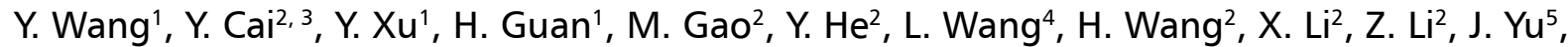 \\ Y. Fu' ${ }^{5}$, Y. Zhang ${ }^{5}$, Y. Zhao ${ }^{5}$, D. Xin ${ }^{5}$ \\ 'School of Postgraduates of Inner Mongolia Medical University, Hohhot, China \\ ${ }^{2}$ Human Anatomy of Inner Mongolia Medical University, Hohhot, China \\ ${ }^{3}$ Digital Medical Centre, School of Basic Medical Sciences, Inner Mongolia Medical University, Hohhot, China \\ ${ }^{4}$ Inner Mongolia International Mongolian Medical Hospital, Hohhot, China \\ ${ }^{5}$ The Second Affiliated Hospital of Inner Mongolia Medical University, China
}

[Received: 25 July 2020; Accepted: 19 August 2020; Early publication date: 2 September 2020]

Background: Based on computed tomography images of the thoracolumbar intervertebral foramen and its surrounding parameters, and analysing the intervertebral foramen morphology and the correlation between the measured parameters, to provide an anatomical basis for clinical minimally invasive transvertebral surgery. Materials and methods: Ten fresh adult cadaveric specimens (32-50 years old) with bilateral $\left(T_{1}-S_{1}\right)$ spinal segments were selected for a total of 20 sides, a total of 340 intervertebral foramens and were measured with vernier callipers in the Department of Anatomy, Inner Mongolia Medical University. The intervertebral foramen height, the minimum sagittal diameter of the foramen, the width of the spinal ganglion, the sagittal diameter of the spinal ganglion and the sagittal diameter of the intervertebral foramen were measured. This study was reviewed and approved by the local Ethics Committee. Results: The results of the minimum sagittal diameter of the $T_{9-10} \sim L_{5} / S$, intervertebral foramen were $(6.93 \pm 1.99) \mathrm{mm},(7.33 \pm 1.44) \mathrm{mm},(7.41 \pm 0.63) \mathrm{mm},(6.85 \pm$ $\pm 1.08) \mathrm{mm},(6.79 \pm 1.86) \mathrm{mm},(7.82 \pm 3.25) \mathrm{mm},(8.23 \pm 2.27) \mathrm{mm},(9.17 \pm 2.33) \mathrm{mm}$, $(8.38 \pm 1.63) \mathrm{mm}$; the average height of the $T 2 / 3$ to $L 5 / S 1$ intervertebral space was $(4.82 \pm 1.88) \mathrm{mm},(3.95 \pm 0.80) \mathrm{mm},(4.04 \pm 0.52) \mathrm{mm},(4.26 \pm 0.78) \mathrm{mm},(4.39 \pm$ $\pm 1.16) \mathrm{mm},(5.15 \pm 1.59) \mathrm{mm},(5.51 \pm 1.49) \mathrm{mm},(5.97 \pm 2.60) \mathrm{mm},(7.13 \pm 2.07) \mathrm{mm}$, $(8.94 \pm 1.37) \mathrm{mm},(9.01 \pm 1.47) \mathrm{mm},(11.63 \pm 1.63) \mathrm{mm},(14.20 \pm 1.37) \mathrm{mm}$, $(14.22 \pm 2.33) \mathrm{mm},(14.22 \pm 2.33) \mathrm{mm},(13.32 \pm 1.37) \mathrm{mm}$ intervertebral foramen height, intervertebral foramen minimum sagittal diameter, spinal ganglion width, spinal ganglion sagittal diameter. $P>0.05$ for comparison of the left and right sides of the intervertebral space, with no statistically significant difference. $L_{4 / 5^{\prime}} L_{5} / S$, segment left and right bilateral contrast with the middle height of the vertebral space $p<0.05$, the difference is statistically significant. The remaining segments left and right bilaterally contrasted $p>0.05$, and the difference was not statistically significant. Conclusions: The minimum height of intervertebral foramen in the thoracolumbar segment was $T_{6 / 7}$ and $L_{1 / 2}$ was the minimum height in the lumbar segment. When placing a spinal endoscopic working channel safely into intervertebral foramen, it is necessary to perform an enlarging foraminoplasty to reduce the risk of injury to the exiting nerve root. (Folia Morphol 2021; 80, 3: 675-682)

Key words: adult, thoracic spine, lumbar spine, intervertebral foramen, anatomical measurements

Address for correspondence: Dr. H. Wang; Dr. X. Li, Human Anatomy Inner Mongolia Medical University Basic Medical College, Hohhot, 10110, China, tel: +864716657009, fax: +864716657562, e-mail: 18647398868@163.com; 798242742@qq.com 


\section{INTRODUCTION}

The nucleus pulposus of the intervertebral disc decreases with age, leading to disc degeneration, which is the major cause of herniated discs. Thoracic disc herniation (TDH) has a low incidence of TDH, which has been reported to account for $0.25-0.75 \%$ of spinal disc herniation, compared to $0.1-0.0001 \%$ in the general population [9]. It mainly occurs between $\mathrm{T}_{8}-\mathrm{L}_{1}$, most often in patients aged $30-50$ years, with an equal proportion of men and women, with complex and varied clinical manifestations, diagnostic difficulties and surgical difficulties [3]. Lumbar disc herniation is a common orthopaedic condition. The prevalence of lower back pain is estimated at $4.8 \%$ in men aged 35 years, and $2.5 \%$ in women of that age. Spinal nerve entrapment due to intervertebral foramen stenosis, or spinal space herniation is more common [2]. For patients who have failed to respond to conservative treatment, surgery is often used, and minimally invasive surgery is used to treat herniated discs through lumbar intervertebral spondylolisthesis. The results are significant, and there is a large body of literature confirming the clinical superiority of the transforaminal path for lumbar disc herniation over traditional surgical approaches $[1,6]$. Treatment of herniated discs using intervertebral endoscopic techniques can reduce peri-spinal muscle and soft tissue injury, increase spinal stability, and is associated with a small trauma, rapid recovery and short hospital stay [8]. With the increasing development of a large number of intervertebral foramen techniques, there is a need for clinicians to further improve their understanding of intervertebral foramen anatomy. In this paper, we use adult cadaveric specimens to study the anatomical and morphological changes of intervertebral foramen in the thoracolumbar spine and to investigate the normal intervertebral foramen, spinal nerves and intervertebral space morphology, measurement of its diameter line, and correlation analysis of the spinal ganglion with the size of the intervertebral foramen. It provides anatomical basis for selection of position and angle for minimally invasive clinical transvertebral foramen path surgery.

\section{MATERIALS AND METHODS}

Ten fresh cadaver specimens were collected, ranging in age from 32 to 50 years, with an average age of 43.2 years, on the intact spine of a normal fresh adult cadavers $\left(T_{1}\right.$ to $\left.S_{1}\right)$, bilaterally on 20 sides with a total of 340 intervertebral foramen. This study was reviewed and approved by the local Ethics Committee. The data in the literature are varied, mainly because of individual variations and the different degrees of degenerative change in the spine. In this study, none of the subjects had symptoms or any degenerative changes of the spine. Therefore, our results provide the normal morphometric anatomy for the nerve roots and dorsal root ganglion.

\section{Observational indicators}

Use of vernier callipers (Japan Mitutoyo, accuracy $0.02 \mathrm{~mm}$ ), compasses, stainless steel rulers (accuracy $1 \mathrm{~mm}$ ), medical power saws. Drill (Shanghai Bojin Medical Instrument Co., Ltd., BYJ-1) tools, surgical instruments: scalpels, surgical scissors, haemostatic forceps, dissecting instruments, etc. forceps, hacksaw. Measure the height (vertical diameter) of the intervertebral foramen bilaterally at each spinal segment with reference to Wu Bo (2017) [13] defining the intervertebral level at the upper margin of the intervertebral space The distance from the anterior boundary of the foramen to the posterior boundary of the intervertebral foramen is the minimum sagittal diameter of the intervertebral foramen, the wide spinal ganglion, the sagittal diameter of the spinal ganglion, and the intervertebral space. Left, middle, and right heights' measurements were taken by the same measurer familiar with the anatomical landmarks for which the relevant indexes were collected, and the recording length $/ \mathrm{mm}(\mathrm{d} / \mathrm{mm})$ was Unit.

Specimen production dissect the thoracic and lumbar segments of 10 adult cadaveric specimens, remove internal organs, and use a hacksaw to saw the upper end of the spinal specimen from the bulge. Horizontal dissociation, marking the $12^{\text {th }}$ rib, identifying the lumbar segment, inferiorly dissociating horizontally from the hip joint, followed by local dissociative manoeuvres, removing the skin The lumbaris major muscle is carefully resected to expose the entire thoracic spine, lumbar spine, intervertebral space, intervertebral foramen, and the muscles and surrounding soft tissue structures. Nerve root structures, keeping the position of nerve roots within the intervertebral foramen intact, and making morphological observations of the intervertebral foramen, nerve roots, and intervertebral spaces (Fig. 1). The upper end of the scalpel was detached from the vertebral space at the $C_{7} / T_{1}$ segment with a scalpel, and the lower end of the scalpel was used to divide the ribs one by one along the rib joint with a medical electric saw drill and the thoracic vertebrae (Fig. 2). The intervertebral foramen height was measured, i.e., the distance from the superior to the inferior margin of the foramen; the minimum sagittal diameter of 


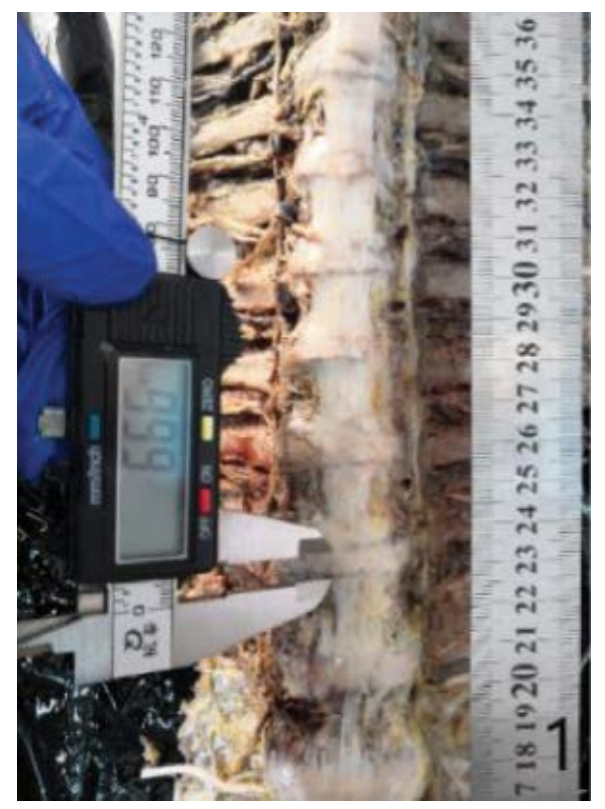

Figure 1. Lumbar intervertebral foramen of the thoracic vertebrae, measuring the height of the intervertebral disc.

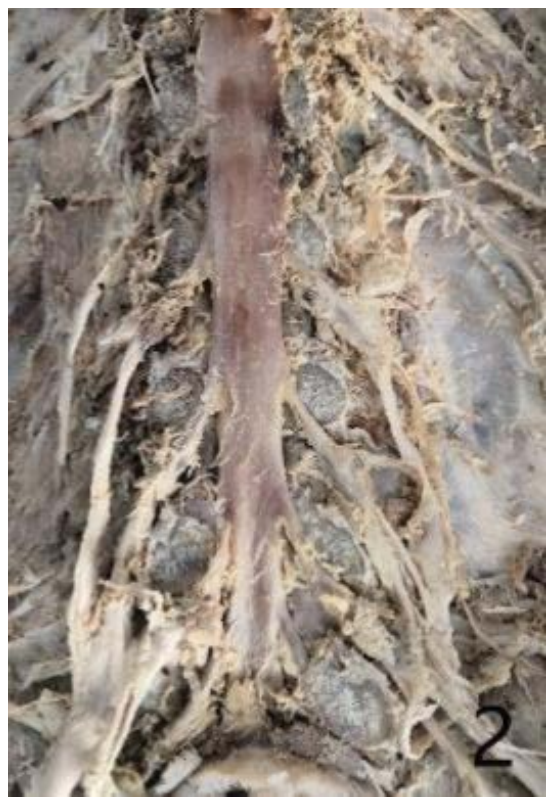

Figure 2. Posterior view of the spinal canal.

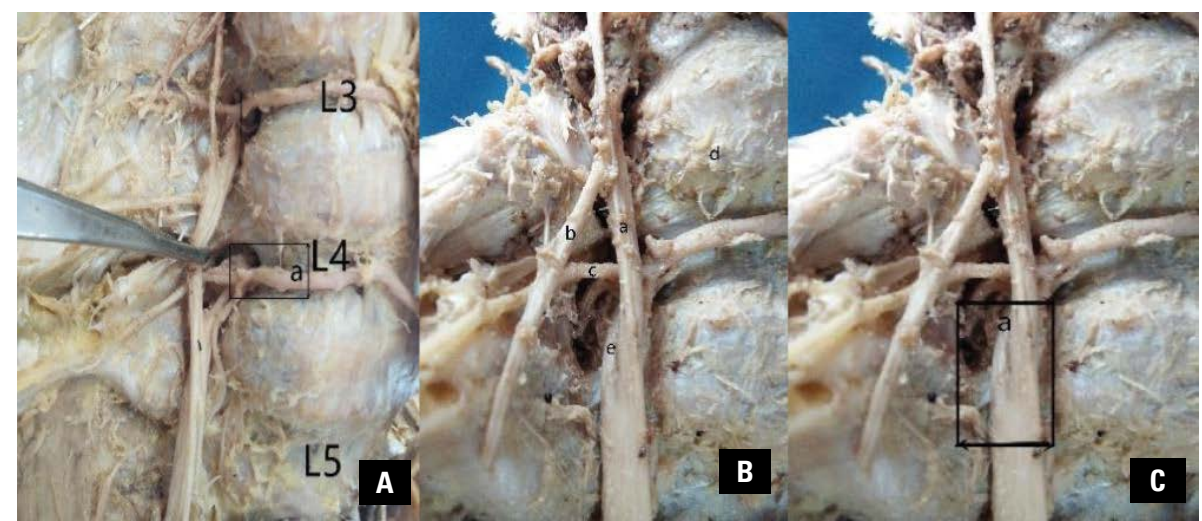

Figure 3. A. Sympathetic dry; B. Grey white traffic branch; C. Lumbar artery; D. Vertebral; E. Pedicle anterior branch.

the foramen, i.e., the distance between the posterior margin of the intervertebral space and the distance; observation of the intervertebral foramen walking nerves (Fig. 3A-C); measurement of the intervertebral space height, i.e., the superior and inferior endplate levels distance between them (Fig. 4); the spinal sagittal diameter, which is the maximum distance between the axial edges of the spinal ganglia; the spinal width, which is the distance perpendicular to the maximum distance between spinal ganglion edges (Fig. 5).

\section{Statistical methods}

GraphPad Prism 8.0 was used and the measures were expressed as mean \pm standard deviation $(\bar{x} \pm s)$. Paired $t$-tests were used between left and right lateral com- parisons and between ipsilateral comparisons; one-way ANOVA was used between comparisons of different segments; determination of intervertebral foramen index and spinal ganglion index by bivariate correlation Pearson's correlation coefficient test the correlation between. Using Pearson's correlation coefficient test, the correlation coefficient $(r)$ is interpreted as follows: $<0.2$ indicates a weak correlation. 0.2-0.4 indicates weak correlation, 0.4-0.7 moderate correlation, 0.7-0.9 high correlation, $>0.9$ indicates almost perfect correlation. The test level was alpha $=0.05 ; p<0.05$ was statistically significant.

\section{RESULTS}

Left and right bilateral comparison $p>0.05$ for the left and right sides of each measure, the differenc- 
es are not statistically significant, and the statistical data are combined. $\mathrm{T}_{1 / 2} \sim \mathrm{L}_{5} / \mathrm{S}_{1}$ segment intervertebral foramen height comparison $p<0.0001$, the difference is statistically significant. $T_{9 / 10} \sim L_{5} / S_{1}$ segment. The minimum sagittal diameter of intervertebral foramen was $p>0.05$, and the difference was not statistically significant. $T_{6 / 7} \sim L_{5} / S_{1}$ segment interspinal

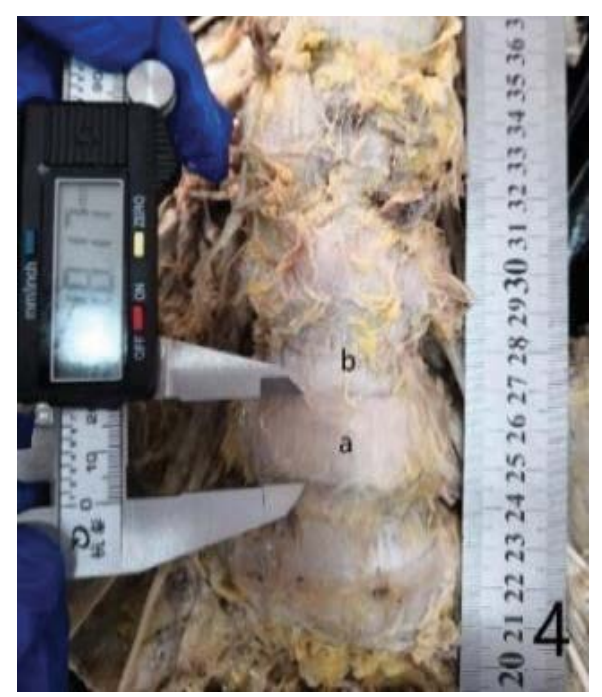

Figure 4. Measuring the right side of the intervertebral disc; a vertebral body; $b$ - intervertebral disc. nerve $(p<0.05)$, statistically significant difference. $P<0.05$ for $T_{7 / 8} \sim L_{5} / S_{1}$ intersegmental chiropractic width. The differences were statistically significant (Tables 1, 2).

Measurement of anatomical parameters of the intervertebral space $p>0.05$ for comparison of the left and right heights of the intervertebral space,

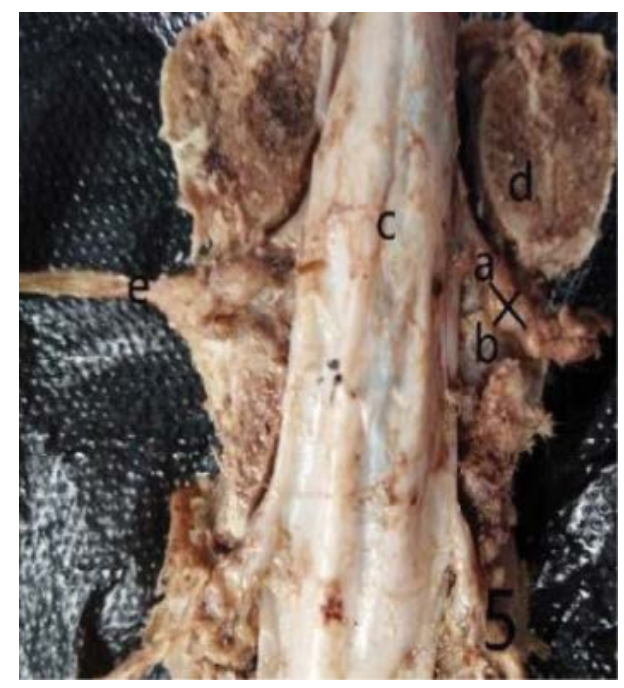

Figure 5. Measurement of spinal ganglion; a - spinal ganglion wide; $b$ - spinal ganglion sagittal diameter; $\mathrm{c}$ - dural sac; $\mathrm{d}$ pedicle; e - spinal nerve posterior root.

Table 1. Measurements of the high of intervertebral foramens, minimal sagittal diameter of intervertebral foramen (mean \pm standard deviation)

\begin{tabular}{|c|c|c|c|c|c|c|c|c|}
\hline & \multicolumn{4}{|c|}{ The high of intervertebral foramens $[\mathrm{mm}](\mathrm{n}=340)$} & \multicolumn{4}{|c|}{ Minimal sagittal diameter of intervertebral foramen $[\mathrm{mm}](\mathrm{n}=\mathbf{1 8 0})$} \\
\hline & Left & Right & Bilateral & $\mathbf{P}$ & Left & Right & Bilateral & $\mathbf{P}$ \\
\hline $\mathrm{T}_{1 / 2}$ & $9.38 \pm 0.70$ & $9.03 \pm 1.10$ & $9.20 \pm 0.91$ & 0.516 & - & - & - & - \\
\hline $\mathrm{T}_{2 / 3}$ & $9.44 \pm 0.83$ & $9.52 \pm 1.39$ & $9.48 \pm 1.06$ & 0.909 & - & - & - & - \\
\hline $\mathrm{T}_{3 / 4}$ & $9.43 \pm 1.55$ & $8.95 \pm 1.73$ & $9.19 \pm 1.55$ & 0.359 & - & - & - & - \\
\hline $\mathrm{T}_{45}$ & $7.29 \pm 1.24$ & $7.51 \pm 1.29$ & $7.40 \pm 1.14$ & 0.799 & - & - & - & - \\
\hline $\mathrm{T}_{5 / 6}$ & $6.26 \pm 1.07$ & $6.09 \pm 1.26$ & $6.17 \pm 1.09$ & 0.754 & - & - & - & - \\
\hline $\mathrm{T}_{6 \pi}$ & $5.49 \pm 1.25$ & $6.35 \pm 1.52$ & $5.92 \pm 1.20$ & 0.471 & - & - & - & - \\
\hline $\mathrm{T}_{7 / 8}$ & $7.28 \pm 3.68$ & $7.69 \pm 3.40$ & $7.48 \pm 3.34$ & 0.504 & - & - & - & - \\
\hline $\mathrm{T}_{8 / 9}$ & $10.01 \pm 5.97$ & $9.00 \pm 6.14$ & $9.46 \pm 5.75$ & 0.006 & - & - & - & - \\
\hline$T_{9 / 10}$ & $9.31 \pm 5.09$ & $9.10 \pm 4.74$ & $9.21 \pm 4.64$ & 0.626 & $6.63 \pm 0.52$ & $7.23 \pm 3.36$ & $6.93 \pm 1.99$ & 0.815 \\
\hline $\mathrm{T}_{10 / 11}$ & $9.84 \pm 3.41$ & $9.85 \pm 3.41$ & $9.71 \pm 3.03$ & 0.071 & $7.22 \pm 1.00$ & $7.44 \pm 0.85$ & $7.33 \pm 1.44$ & 0.792 \\
\hline$T_{11 / 12}$ & $10.44 \pm 1.68$ & $10.57 \pm 1.74$ & $10.51 \pm 1.73$ & 0.842 & $7.40 \pm 0.56$ & $7.42 \pm 0.82$ & $7.41 \pm 0.63$ & 0.976 \\
\hline $\mathrm{T}_{12 /} \mathrm{L}_{1}$ & $9.00 \pm 1.07$ & $10.23 \pm 1.68$ & $9.50 \pm 1.41$ & 0.371 & $7.38 \pm 1.01$ & $6.32 \pm 0.83$ & $6.85 \pm 1.08$ & 0.104 \\
\hline $\mathrm{L}_{1 / 2}$ & $12.22 \pm 2.10$ & $12.32 \pm 1.52$ & $12.27 \pm 1.34$ & 0.854 & $7.48 \pm 2.35$ & $6.00 \pm 1.29$ & $6.79 \pm 1.86$ & 0.218 \\
\hline$L_{2 / 3}$ & $14.71 \pm 2.29$ & $15.01 \pm 1.26$ & $14.86 \pm 2.36$ & 0.799 & $8.70 \pm 3.90$ & $6.94 \pm 2.56$ & $7.82 \pm 3.25$ & 0.468 \\
\hline$L_{3 / 4}$ & $16.68 \pm 2.27$ & $15.21 \pm 2.53$ & $15.94 \pm 2.43$ & 0.435 & $8.83 \pm 2.93$ & $7.62 \pm 1.241$ & $8.23 \pm 2.27$ & 0.317 \\
\hline $\mathrm{L}_{4 / 5}$ & $15.44 \pm 2.23$ & $15.40 \pm 2.75$ & $15.42 \pm 2.34$ & 0.964 & $9.02 \pm 2.61$ & $9.30 \pm 2.22$ & $9.17 \pm 2.33$ & 0.789 \\
\hline$L_{5} S_{1}$ & $13.37 \pm 2.02$ & $12.40 \pm 2.06$ & $12.88 \pm 2.13$ & 0.194 & $8.85 \pm 2.07$ & $7.92 \pm 1.78$ & $8.38 \pm 1.63$ & 0.128 \\
\hline F-value & \multicolumn{2}{|c|}{11.27} & $P<0.0001$ & & \multicolumn{2}{|c|}{1.91} & $P>0.05$ & \\
\hline
\end{tabular}


Table 2. The transverse diameter of the dorsal root ganglion $(n=240)$ and longitudinal diameter of the dorsal root ganglion $(n=220)$ (mean \pm standard deviation)

\begin{tabular}{|c|c|c|c|c|c|c|c|c|}
\hline & \multicolumn{4}{|c|}{ The transverse diameter of the dorsal root ganglion [mm] } & \multicolumn{4}{|c|}{ Longitudinal diameter of the dorsal root ganglion [mm] } \\
\hline & Left & Right & Bilateral & $\mathbf{P}$ & Left & Right & Bilateral & $\mathbf{P}$ \\
\hline $\mathrm{T}_{6 / 7}$ & $5.03 \pm 1.80$ & $4.79 \pm 1.49$ & $4.91 \pm 1.48$ & 0.556 & - & - & - & - \\
\hline $\mathrm{T}_{7 / 8}$ & $4.38 \pm 0.85$ & $4.39 \pm 1.18$ & $4.38 \pm 0.92$ & 0.984 & $6.22 \pm 0.35$ & $7.33 \pm 1.00$ & $6.95 \pm 0.95$ & 0.251 \\
\hline $\mathrm{T}_{8 / 9}$ & $4.94 \pm 1.90$ & $5.10 \pm 2.00$ & $5.02 \pm 1.75$ & 0.216 & $7.35 \pm 1.42$ & $6.56 \pm 0.22$ & $6.96 \pm 1.03$ & 0.283 \\
\hline $\mathrm{T}_{9 / 10}$ & $5.05 \pm 1.52$ & $4.74 \pm 1.67$ & $4.90 \pm 1.51$ & 0.068 & $7.13 \pm 0.93$ & $6.10 \pm 1.21$ & $6.71 \pm 1.05$ & 0.467 \\
\hline $\mathrm{T}_{10 / 11}$ & $5.87 \pm 1.38$ & $5.46 \pm 1.65$ & $5.66 \pm 1.42$ & 0.433 & $8.37 \pm 2.53$ & $7.01 \pm 1.07$ & $7.69 \pm 1.94$ & 0.384 \\
\hline $\mathrm{T}_{11 / 12}$ & $5.77 \pm 1.13$ & $5.46 \pm 0.81$ & $5.61 \pm 1.41$ & 0.647 & $7.61 \pm 1.24$ & $7.03 \pm 1.14$ & $7.31 \pm 1.07$ & 0.675 \\
\hline $\mathrm{T}_{12} \mathrm{~L}_{1}$ & $5.96 \pm 1.38$ & $7.66 \pm 1.65$ & $6.64 \pm 2.18$ & 0.433 & $8.37 \pm 1.53$ & $7.01 \pm 1.07$ & $6.52 \pm 1.24$ & 0.384 \\
\hline $\mathrm{L}_{1 / 2}$ & $6.65 \pm 2.10$ & $6.37 \pm 2.56$ & $6.51 \pm 1.24$ & 0.602 & $6.50 \pm 1.55$ & $6.29 \pm 1.51$ & $7.67 \pm 1.80$ & 0.806 \\
\hline $\mathrm{L}_{2 / 3}$ & $6.42 \pm 2.26$ & $6.80 \pm 2.47$ & $6.61 \pm 2.70$ & 0.472 & $9.31 \pm 1.21$ & $7.80 \pm 1.41$ & $8.55 \pm 1.64$ & 0.499 \\
\hline $\mathrm{L}_{3 / 4}$ & $8.15 \pm 4.11$ & $7.77 \pm 4.72$ & $7.96 \pm 4.18$ & 0.681 & $12.01 \pm 2.20$ & $10.37 \pm 1.70$ & $11.19 \pm 1.14$ & 0.207 \\
\hline $\mathrm{L}_{4 / 5}$ & $8.99 \pm 4.41$ & $9.20 \pm 6.10$ & $9.08 \pm 4.71$ & 0.632 & $11.46 \pm 1.54$ & $9.43 \pm 1.36$ & $10.82 \pm 1.09$ & 0.343 \\
\hline$L_{5 / S 1}$ & $4.65 \pm 0.46$ & $4.24 \pm 0.86$ & $4.44 \pm 0.70$ & 0.198 & $10.41 \pm 1.61$ & $11.1 \pm 1.81$ & $10.46 \pm 1.49$ & 0.144 \\
\hline F-value & \multicolumn{2}{|c|}{3.494} & $P<0.05$ & & \multicolumn{2}{|c|}{3.068} & $P<0.05$ & \\
\hline
\end{tabular}

Table 3. Intervertebral disc height $[\mathrm{mm}](\mathrm{n}=320)$ (mean \pm standard deviation)

\begin{tabular}{|c|c|c|c|c|c|c|}
\hline & Left & Right & P1 & Middle & Bilateral & P2 \\
\hline$T_{2 / 3}$ & $3.80 \pm 1.37$ & $3.67 \pm 0.58$ & 0.851 & $5.78 \pm 2.11$ & $4.82 \pm 1.88$ & 0.256 \\
\hline $\mathrm{T}_{3 / 4}$ & $3.90 \pm 0.62$ & $3.62 \pm 0.60$ & 0.724 & $4.41 \pm 1.05$ & $3.95 \pm 0.8$ & 0.388 \\
\hline $\mathrm{T}_{4 / 5}$ & $3.92 \pm 0.38$ & $3.71 \pm 0.49$ & 0.657 & $4.49 \pm 0.38$ & $3.99 \pm 0.51$ & 0.1 \\
\hline $\mathrm{T}_{5 / 6}$ & $4.01 \pm 0.50$ & $4.00 \pm 0.84$ & 0.974 & $4.79 \pm 0.84$ & $4.26 \pm 0.78$ & 0.268 \\
\hline $\mathrm{T}_{6 / 7}$ & $4.03 \pm 0.91$ & $4.86 \pm 1.30$ & 0.072 & $4.27 \pm 1.39$ & $4.39 \pm 1.16$ & 0.868 \\
\hline $\mathrm{T}_{7 / 8}$ & $5.48 \pm 2.07$ & $5.28 \pm 0.73$ & 0.88 & $4.82 \pm 1.15$ & $5.21 \pm 1.5$ & 0.651 \\
\hline $\mathrm{T}_{8 / 9}$ & $5.25 \pm 2.21$ & $5.92 \pm 1.08$ & 0.634 & $4.93 \pm 1.23$ & $5.51 \pm 1.49$ & 0.497 \\
\hline$T_{9 / 10}$ & $6.40 \pm 4.05$ & $6.17 \pm 2.47$ & 0.824 & $4.84 \pm 1.82$ & $6.61 \pm 2.87$ & 0.3 \\
\hline $\mathrm{T}_{10 / 11}$ & $6.34 \pm 1.83$ & $8.66 \pm 1.65$ & 0.148 & $6.42 \pm 2.55$ & $7.53 \pm 1.84$ & 0.421 \\
\hline $\mathrm{T}_{11 / 12}$ & $9.70 \pm 0.61$ & $8.83 \pm 0.81$ & $0.003^{*}$ & $8.62 \pm 1.02$ & $8.94 \pm 1.37$ & 0.674 \\
\hline$T_{122} L_{1}$ & $9.29 \pm 1.54$ & $8.94 \pm 1.12$ & 0.551 & $8.81 \pm 1.89$ & $9.01 \pm 1.47$ & 0.787 \\
\hline $\mathrm{L}_{1 / 2}$ & $13.39 \pm 3.00$ & $10.06 \pm 2.32$ & 0.229 & $11.45 \pm 1.67$ & $11.63 \pm 1.63$ & 0.888 \\
\hline$L_{2 / 3}$ & $15.27 \pm 2.53$ & $13.13 \pm 2.38$ & 0.059 & $14.19 \pm 2.37$ & $14.18 \pm 2.15$ & 0.993 \\
\hline $\mathrm{L}_{3 / 4}$ & $14.56 \pm 2.73$ & $12.83 \pm 1.85$ & 0.236 & $15.26 \pm 2.21$ & $13.69 \pm 2.35$ & 0.293 \\
\hline $\mathrm{L}_{4 / 5}$ & $13.72 \pm 2.06$ & $14.40 \pm 0.70$ & 0.558 & $18.22 \pm 2.21$ & $14.06 \pm 1.47$ & $0.002^{*}$ \\
\hline $\mathrm{L}_{5 / \mathrm{S} 1}$ & $13.47 \pm 1.54$ & $13.16 \pm 1.34$ & 0.631 & $20.00 \pm 0.82$ & $13.32 \pm 1.37$ & $0.000^{*}$ \\
\hline
\end{tabular}

${ }^{*} \mathrm{P}<0.05$ - the difference is statistically significant; P1 — for left-right side comparison; P2 — for middle and bilateral comparison

the difference was not statistically significant, and statistical data were combined. $L_{4 / 5}, L_{5} / S_{1} p<0.05$ for the comparison between the left and right sides of the $S_{1}$ segment and the middle height of the intervertebral space, the difference was statistically significant. The remaining segments left and right bilateral and vertebral space intermediate height contrast $p>0.05$, the difference was not statistically significant (Table 3).

Correlation analysis of spinal ganglion width and spinal ganglion sagittal diameter with intervertebral foramen height and intervertebral foramen 


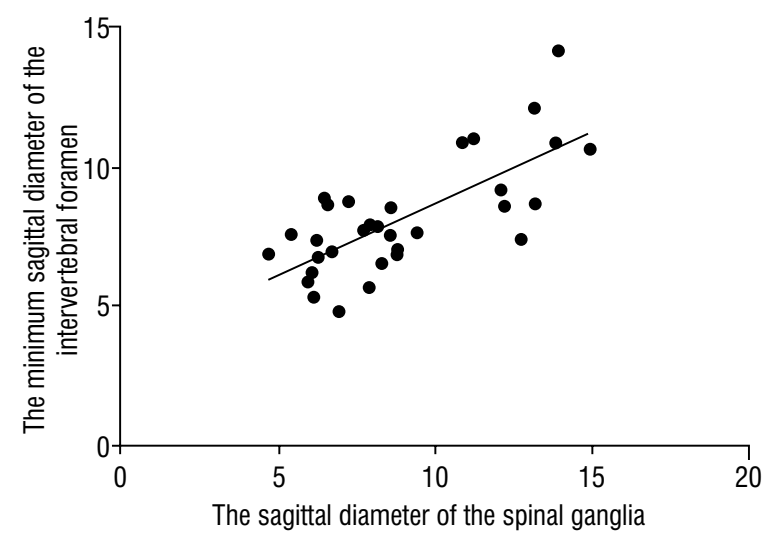

Figure 6. The minimum sagittal diameter of the intervertebral foramen is positively correlated with the sagittal diameter of the spinal ganglia.

minimum sagittal diameter, spinal ganglion sagittal diameter. The minimum sagittal diameter of the intervertebral foramen $(r=0.728)$, with $r$ between 0.7 and 0.9 , positively correlated and highly correlated (Fig. 6).

Analysis of the correlation between intervertebral foramen height and the left, right, and median height of the intervertebral space (Fig. 7). The intervertebral foramen height is positively correlated with the left and right bilateral height of the intervertebral space $(r=0.917)$, with $r$ between 0.7 and 0.9 , and height correlation. The intervertebral foramen height is positively and highly correlated with the median height of the intervertebral space $(r=0.877)$, with $r$ ranging from 0.7 to 0.9 .

\section{DISCUSSION}

There are broadly two methods for measuring intervertebral foramen morphology in China: one is direct measurement and the other is indirect measurement. The direct measurement tool is used to collect data from cadaveric specimens, while the indirect measurement mainly uses $\mathrm{X}$-ray technique, three-dimensional computed tomography imaging and magnetic resonance imaging measurement [4].
In addition, after software post-processing to reconstruct the intervertebral foramen image, the measurement is performed, while the presence of soft tissue shadowing affects the experiment using $X$-ray and magnetic resonance imaging. In this paper, we collected 10 fresh cadaveric specimens and directly measured the morphology of the intervertebral foramen, which enabled direct, realistic and accurate observation of the intervertebral foramen. Zi-xuan et al. [16] measured cadavers under the $X$-ray technique similar to the measurements in this paper. The resultant anterior disc heights were $(11.8 \pm 1.2) \mathrm{mm},(13.0 \pm$ $\pm 1.6) \mathrm{mm},(13.6 \pm 1.9) \mathrm{mm},(14.3 \pm 2.0) \mathrm{mm},(14.7 \pm$ $\pm 2.5) \mathrm{mm}$. This the study is consistent with previous literature and the results are reliable and accurate. Rühli et al. [10] measured cadaveric specimens using vernier callipers. Comparing men and women revealed that the width of the intervertebral foramen was larger in women than in men at the lumbar segment. All specimens in this study were male, and the difference between the left and right sides of each measurement comparing the same individual was not statistically significant.

The bony border morphology of the intervertebral foramen has been described in the literature as an inverted teardrop, inverted pear shape, or oval shape, affecting the intervertebral foramen. There are many factors that contribute to size, such as degenerative changes in the bony borders of the intervertebral foramen, herniated discs, subluxation of the small joints, and hypertrophy of the ligamentum flavum [10]. Zhu et al. [15] retrospectively analysed the changes in intervertebral foramen morphology before and after unilateral transverse lumbar interbody fusion. Compared to the oblique or transverse cage approach, the transverse approach is preferred to reduce lumbar lordosis without affecting the contralateral side. The size of intervertebral foramen is good for decompression of lumbar spinal stenosis, and it is easy to determine the normal size of intervertebral foramen stenosis. The study observed that

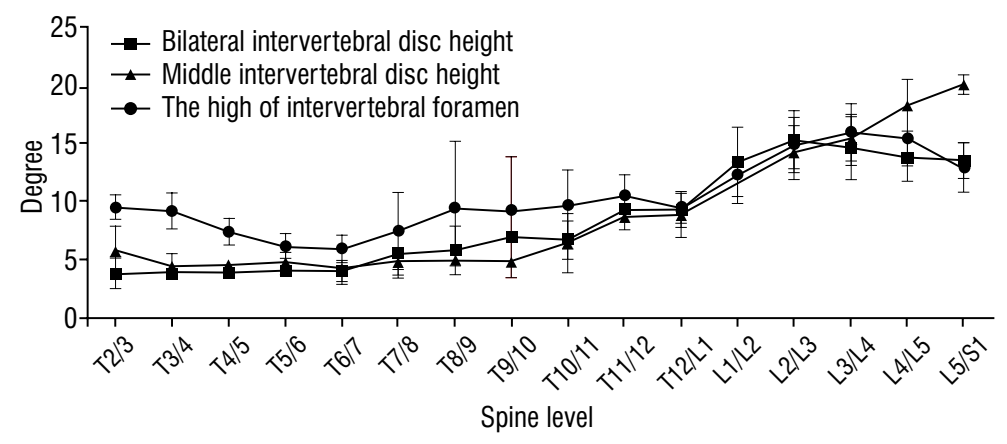

Figure 7. High correlation analysis between the height of intervertebral foramen and the left and right sides and the middle of intervertebral space. 
the transverse section of the spinal nerve root was oval in shape, the transverse section of the thoracic spinal nerve was smaller, and the intervertebral foramen went outward and backward, and the lumbar section was smaller. The spinal nerve cross-section is large and goes obliquely outward and downward after exiting the intervertebral foramen. The wide thoracic segment of the spinal ganglion, $\mathrm{T}_{6}-\mathrm{T}_{12}$, has a gradually increasing trend; the lumbar segment, $\mathrm{L}_{1}-\mathrm{S}_{1}$, has an essentially unchanged trend of $\mathrm{L}_{1 / 2}$; $L_{3 / 4}$ maximal, $L_{5} / S_{1}$ minimal. The trend of the sagittal diameter of spinal ganglion was not obvious in the thoracic segment from $T_{7}$ to $T_{12}$, but in the lumbar segment from $L_{1}$ to $S_{1}, I_{4 / 5}$ maximal, $L_{5} / S_{1}$ minimal. The height of the intervertebral gap was larger in the middle and smaller in the left and right sides of the $\mathrm{L}_{5} / \mathrm{S}_{1}$ and $\mathrm{L}_{4 / 5}$ segments compared to the right and left sides of the $L_{5} / S_{1}$ and $L_{4 / 5}$ segments. Because the intervertebral space morphology dictates that artificial intervertebral space designs cannot be designed to be directly rectangular [11], this study also found that the intervertebral gap height median height in the $L_{5} / S_{1}$ and $L_{4 / 5}$ segments compared to the right and left sides of the spine. Large in the middle and small on the left and right side need to be designed to have a large middle height of the vertebral space and a small height of the vertebral space on both sides, which can improve the biomechanics of the spine.

There are now five types of surgical access for the treatment of $\mathrm{TDH}$, including anterior access, lateral access, pedicle access, intervertebral access, thoracoscopic access. According to the literature, anterior surgical access is more difficult to learn and has a higher incidence of complications [9]. When encountering large disc size, disc calcification and central TDH, the intervertebral approach is not applicable, and there is a lack of treatment in the current literature. Evidence for the safety of surgical access for TDH is attributed to, among other reasons, the low incidence of TDH. Currently, percutaneous endoscopic lumbar discectomy has become the most promising treatment of lumbar intervertebral disc protrusion and minimally invasive spinal surgery. Percutaneous endoscopic lumbar discectomy is widely used in clinical practice with its advantages of less trauma, quicker recovery, shorter operation time, less pain, and improved lumbar spinal cord function [5]. Hoyland et al. [7] found that neural tissue accounted for less than $35 \%$ of the total intervertebral foramen. Other studies found the largest at $\mathrm{L}_{5} / \mathrm{S}_{1}$ and the smallest at $\mathrm{L}_{1 / 2}$. On the contrary, the
$L_{5} / S_{1}$ intervertebral foramen was the smallest. In the study of Rühli et al. [10], osseous intervertebral foramen at the $L_{5}$ level was found to be the largest. The value of the intervertebral foramen sagittal diameter is less than the $L_{1}$ level. The size of the working trocar used for minimally invasive interbody surgery is chosen to be less than the height of the intervertebral space measured in this paper, and the outer diameter of the annular saw is often chosen to be $7.5 \mathrm{~mm}$ [14], without articular projection enlargement, in intervertebral foraminoscopy. The operative space is small, and the lower intervertebral foramen is relatively safe compared to the upper, due to the small number of vital structures. The YESS technique requires Kambin triangle entry, this paper found that the spinal ganglion sagittal diameter is positively correlated with the minimum sagittal diameter of the intervertebral foramen, and careful attention should be paid during surgery. Identify the structures surrounding the intervertebral foramen to avoid damage to the surrounding spinal nerves. The TEYESS requires clipping of the supra-articular foramina to enlarge the intervertebral foramen without entering the intervertebral space through Kambin's triangle. The trocar is more easily accessed by the enlarged intervertebral foramen.

In addition, $T_{1 / 2}$ to $L_{5} / S_{1}$ intervertebral foramen height observes that the thoracic intervertebral foramen height changes in $T_{2}$ to $T_{12}$ with a trend of decreasing first. The trend of change from $T_{2 / 3}$ to $\mathrm{L}_{5} / \mathrm{S}_{1}$ in the thoracic and lumbar segments is that the first one gradually increases, the second one increases after $T_{6 / 7}$, and the third one is the smallest in $T_{11 / 12}$. Decreasing to $T_{6 / 7}$ minimum, then increasing to $T_{7 / 8}$ greater, then decreasing, $T_{9 / 10}$ smaller, then increasing, reaching larger at $\mathrm{L}_{3 / 4}$. Lumbar intervertebral foramen height is greater at $\mathrm{L}_{3 / 4}, \mathrm{~L}_{4 / 5}$, smallest at $\mathrm{L}_{1 / 2}$, smaller at $L_{5} / S_{1}$; lumbar $L_{1}-S_{1}$ the minimum sagittal diameter of the intervertebral foramen tends to increase and then decrease, being smallest at $L_{1 / 2}$ and largest at $\mathrm{L}_{4 / 5}$. Vialle et al. [12] measured the lumbar segments of eight male adult specimens. The mean value of the maximum longitudinal diameter of the spinal ganglion was $13.25 \mathrm{~mm}$ and the mean value of the minimum sagittal diameter of the spinal ganglion was $7.05 \mathrm{~mm}$, which is similar to that of this paper. The findings were consistent. The intervertebral foramen height in the same segment was greater than the minimum sagittal diameter of the intervertebral foramen. The minimum sagittal diameter of intervertebral foramen in different segments is not statistical- 
ly significant, and the influence of different vertebral sequence changes can be ignored when designing. Knowing the change of intervertebral foramen in different segments of the thoracolumbar spine and choosing the correct intervertebral perforator trocar will help to improve the success rate of surgery and reduce the number of cases.

\section{CONCLUSIONS}

The minimum height of intervertebral foramen in the thoracolumbar segment was $\mathrm{T}_{6 / 7}$ and $\mathrm{L}_{1 / 2}$ was the minimum height in the lumbar segment. When placing a spinal endoscopic working channel safely into intervertebral foramen, it is necessary to perform an enlarging foraminoplasty to reduce the risk of injury to the exiting nerve root.

\section{Acknowledgements}

This study was supported by Study on the National Natural Science Foundation of China (81860383, 81560348, 81460330); Natural Science Foundation of Inner Mongolia Autonomous Region (2020MS08124); Young Science and Technology Talents Project of Inner Mongolia Education Department (njyt-15-b05); Science and Technology Plan Project of Inner Mongolia Autonomous Region (2016,2019GG115); Science and Technology Innovation Guidance Project of Inner Mongolia Autonomous Region (2017); Natural Science Foundation of Inner Mongolia Autonomous Region (2016ms08131,2020MS08124); Inner Mongolia Scholarship Fund, Department of Human Resources and Social Services (TDK2017KJBW012); Inner Mongolia Autonomous Region "Grassland Talent" project youth innovation and entrepreneurship talent project (2020); Transformation Project of Scientific and Technological Achievements of Inner Mongolia Medical University (201903); Inner Mongolia Mongolian Medicine Collaborative Innovation Centre (2021, No.MyyxTYB202104).

\section{Conflict of interest: None declared}

\section{REFERENCES}

1. Ahn SS, Kim SH, Kim DW, et al. Comparison of outcomes of percutaneous endoscopic lumbar discectomy and open lumbar microdiscectomy for young adults: a retrospective matched cohort study. World Neurosurg. 2016; 86: 250-258, doi: 10.1016/j.wneu.2015.09.047, indexed in Pubmed: 26409086.

2. Bono C. Lumbar disc herniation and radiculopathy. Principles Orthopedic Practice Primary Care Providers. 2017: 37-46, doi: 10.1007/978-3-319-68661-5 3.
3. Court C, Mansour E, Bouthors C. Thoracic disc herniation: Surgical treatment. Orthop Traumatol Surg Res. 2018; 104(1S): S31-S40, doi: 10.1016/j.otsr.2017.04.022, indexed in Pubmed: 29225115.

4. Cramer GD, Cantu JA, Dorsett RD, et al. Dimensions of the lumbar intervertebral foramina as determined from the sagittal plane magnetic resonance imaging scans of 95 normal subjects. J Manipulative Physiol Ther. 2003; 26(3): 160-170, doi: 10.1016/S0161-4754(02)54109-9, indexed in Pubmed: 12704308.

5. Dalbayrak S, Yaman O, Yilmaz M, et al. Transforaminal approach in lumbar disc herniations: transforaminal microdiscectomy (TFMD) technique. Turk Neurosurg. 2015; 25(1): 29-35, doi: 10.5137/1019-5149.JTN.8197-13.1, indexed in Pubmed: 25640542.

6. He S, Sun Z, Wang Y, et al. Combining YESS and TESSYS techniques during percutaneous transforaminal endoscopic discectomy for multilevel lumbar disc herniation. Medicine (Baltimore). 2018; 97(28): e11240, doi: 10.1097/ MD.0000000000011240, indexed in Pubmed: 29995758.

7. Hoyland JA, Freemont AJ, Jayson MI. Intervertebral foramen venous obstruction. A cause of periradicular fibrosis? Spine (Phila Pa 1976). 1989; 14(6): 558-568, doi: 10.1097/00007632198906000-00002, indexed in Pubmed: 2749370.

8. Kanno H, Aizawa T, Hahimoto Ko, et al. Minimally invasive discectomy for lumbar disc herniation: current concepts, surgical techniques, and outcomes. Int Orthop. 2019; 43(4): 917-922, doi: 10.1007/s00264-018-4256-5, indexed in Pubmed: 30607499.

9. Kerezoudis P, Rajjoub KR, Goncalves S, et al. Anterior versus posterior approaches for thoracic disc herniation: Association with postoperative complications. Clin Neurol Neurosurg. 2018; 167: 17-23, doi: 10.1016/j.clineuro.2018.02.009, indexed in Pubmed: 29428625.

10. Rühli FJ, Müntener M, Henneberg M. Human osseous intervertebral foramen width. Am J Phys Anthropol. 2006; 129(2): 177-188, doi: 10.1002/ajpa.20263, indexed in Pubmed: 16323199.

11. Tang R, Gungor C, Sesek RF, et al. Morphometry of the lower lumbar intervertebral discs and endplates: comparative analyses of new MRI data with previous findings. Eur Spine J. 2016; 25(12): 4116-4131, doi: 10.1007/s00586016-4405-8, indexed in Pubmed: 26873104.

12. Vialle $E$, Vialle LR, Contreras W, et al. Anatomical study on the relationship between the dorsal root ganglion and the intervertebral disc in the lumbar spine. Rev Bras Ortop. 2015; 50(4): 450-454, doi: 10.1016/j.rboe.2015.06.013, indexed in Pubmed: 26401504.

13. Wu Bo, Zhao qing-hao, Zhou Xiao-qi, et al. Applied anatomy of lumbar intervertebral foramen mirror. Chinese J Clin Anat. 35.01; 2017; 5-8, doi: 10.13418/j.issn.1001165x.2017.01.002

14. Zhen-zhou L, Wen-wen W, Shu-xun H. Design and clinical application of the instrument for percutaneous posterolateral lumbar foraminoplasty. Chinese J Orthopaedics. 2011; 10: 1026-1032, doi: CNKI:SUN:ZHGK.0.2011-10-011.

15. Zhu K, Yan S, Guo S, et al. Morphological changes of contralateral intervertebral foramen induced by cage insertion orientation after unilateral transforaminal lumbar interbody fusion. J Orthop Surg Res. 2019; 14(1): 79, doi: 10.1186/ s13018-019-1121-1, indexed in Pubmed: 30866988.

16. Zi-xuan W, Tao S. Parameter measurement of lumbar disc related to artificial disc replacement in Chinese. J Clin Rehab Tissue Engineering Res. 2011; 15(48): 8973-8976, doi: CNKI:SUN:XDKF.0.2011-48-022. 